\section{In Sorge um die Homöopathie}

\author{
L. Fäh
}

\begin{abstract}
Aktueller Stand
Die treffende Karikatur von ANNA [1] betreffend die Zukunft der Homöopathie hat mich bewogen, einige Gedanken und Bedenken zu äussern.

Seit dem 1. Juli 1999 ist die Homöopathie eine Pflichtleistung der Krankenkassen; wer als homöopathischer Arzt über die Grundversicherung abrechnen will, muss einen Fähigkeitsausweis in "klassischer Homöopathie» besitzen und eine jährliche obligatorische Fortbildung absolvieren. Welche Fähigkeiten sind denn nun gefragt, und welche Fortbildung muss besucht werden, nach dem sich die Homöopathie weltweit in einem Zustand völliger Orientierungslosigkeit [2] befindet, immer mehr ärztliche Kollegen mitten in ihrer Ausbildung zum homöopathischen Arzt diese abbrechen und der Methode wegen fehlender Erfolge den Rücken kehren?

Um des Lesers Zeit nicht unnötig zu beanspruchen, wurden in dieser Arbeit Textstellen, deren Lektüre in erster Linie für homöopathisch tätige Ärzte von Interesse sind, farbig unterlegt.
\end{abstract}

\section{Was ist «Homöopathie»?}

Mit Blick auf die Therapiegeschichte lässt sich diese Frage zufriedenstellend beantworten; ich zitiere aus einer auf Quellenstudien beruhenden medizinhistorisch anerkannten Arbeit [3] unter dem Stichwort "Homöopathie»:

[griech.] vom dt. Arzt S. Hahnemann (1755-1843) begründetes Heilverfahren ...

Kommentar: Der Begründer des Heilverfahrens war demnach Arzt und nicht Heilpraktiker; eine klarere Abgrenzung der homöopathischen Ärzte ist somit ein Gebot der Stunde, denn: «seit Hahnemann seine weltweit bekannt gewordene Lehre ‘Homöopathies genannt hat, nennen sich Ärzte, aber auch Nicht-Ärzte

Korrespondenz:

Dr. med. L. Fäh

FMH Innere Medizin

Solothurnstrasse 10

CH-2543 Lengnau aller Art, die sie zu lehren und zu praktizieren vorgeben, «Homöopathen. Wer sich jedoch um Hahnemanns authentische Lehre bemüht, muss konstatieren, dass sich die sog. Homöopathen von heute in ihrer überwiegenden Mehrheit von Hahnemanns Denken und Wirken als Arzt noch weit mehr entfernt haben, als manche ihrer Vorgänger schon zu Hahnemanns Zeit.» [4] Soweit der ehemalige Präsident des "Schweizerischen Vereins homöopathischer Ärzte», Dr. W. Buschauer.

Hahnemann forderte zeitlebens, dass nur Ärzte die Homöopathie ausüben dürfen und dass die homöopathischen Ärzte stets auch auf dem höchsten Stand des Wissens der Medizin allgemein sein müssen; dies, weil jeder ethisch verpflichtete Arzt in der Lage sein müsse, dem Patienten das vielversprechendste Heilverfahren zu empfehlen. Hahnemann war ein Eklektiker in der Therapie (nicht jedoch in der Theorie) [5]. Die Homöopathie ist zudem eine reine Therapiemethode und in keiner Art und Weise eine diagnostische Methode.

Für homöopathische Ärzte: Das Stellen einer "Arzneimitteldiagnose» entbindet uns nicht von der Verpflichtung, zuerst, wenn immer möglich, eine Diagnose zu stellen; "Arzneimitteldiagnosen» entsprechen einer nicht zulässigen Umgehung dieses Grundsatzes und stellen eine oft zu vereinfachende, z.T. der Symbolik entlehnte Anthropomorphisierung dar etc.

"Weltanschaulicher Hintergrund ist das Gedankengut der Aufklärung; Hahnemann stand dem frz. Vitalismus und der Teleologie nahe ..." [3].

Kommentar: Dies bedeutet u. a., dass Hahnemann in scharfem Gegensatz zur deutschen Romantik in der Medizin stand, einer Romantik, die eine in der Tradition Paracelsus' stehende Mystik und Symbolik weiterspann.

Für homöopathische Ärzte: Wer demnach heute, 1999, in der Homöopathie eine gelbe Pflanze bei Ikterus oder ein Schlangengift (Kaltblüter) bei kälteempfindlichen Patienten zum Einsatz bringen will, steht kaum in der Tradition Hahnemanns [2]. Das gleiche gilt für Homöopathen, die Arzneimittel aufgrund des Periodensystems verordnen [6] oder wenn «künstlerische Homöopathie» wie folgt (nach Sankaran bzw. Vithoulkas) angewendet wird, wenn es z.B. heisst «... wenn ein Patient wie ein Schwein aussieht, muss seine Arznei ein tierisches Mittel sein, aber da wir keine Arznei haben, die vom Schwein stammt, könnten wir das nächst analoge Mittel, nämlich ... den Frosch, also Bufo, verschreiben, selbst wenn die Symptome des Patienten Baryta carbonicum anzeigen mögen». [2] 
"Die Homöopathie ist die Summe der Lehren und Grundsätze der theoretischen und praktischen homöopathischen Heilkunst. Mehrere Regeln und Modellvorstellungen stehen gleichberechtigt nebeneinander, einige wurden von Hahnemann selbst im Lauf der Zeit in ihrer Bedeutung eingeschränkt, wie etwa die bekannte Simile-Regel (das Behandeln von Krankheitszuständen mit Arzneimitteln, welche bei der Prüfung am Gesunden ähnliche Symptome hervorzubringen mögen - similia similibus).» [3]

Kommentar: Wer glaubt, diesen Aussagen widersprechen zu müssen, da er wähnt, sie bei Hahnemann in dessen Originalwerken nicht finden zu können, dem sei dringend empfohlen, das Werk eines der begabtesten Schüler Hahnemanns, nämlich G. H. G. Jahrs, zu lesen [7].

"Als Heilmittel übernahm Hahnemann den Arzneimittelschatz früherer Ärztegenerationen (Pflanzen, Mineralien, Tier- und später Krankheitsprodukte). Er half damit, diesen über die Zeit des therapeutischen Nihilismus (in der Mitte des 19. Jahrhunderts) zu retten. Grundpfeiler der Homöopathie ist der sogenannte rempirische Krankheitsbegriff. Dieser besteht in der Erkenntnis, dass die Krankheit an sich nicht erkennbar ist; die zugrundeliegende Krankheit gibt sich lediglich durch die Krankheitszeichen und Symptome zu erkennen. Zudem sind alle Krankheitsursachen stets äusserer Natur (Krankheitserreger, Verletzung, Bausteinmangel etc.)." [3]

Kommentar: Entscheidender Unterschied zwischen "Schulmedizin" und Homöopathie ist demnach der Krankheitsbegriff: In der "Schulmedizin" ist er uneinheitlich; es werden dort neben dem anatomischen/ zellularpathologischen Krankheitsbegriff (nach Virchow) auch diejenigen akzeptiert, welche vorwiegend in der Psychiatrie Anwendung finden und die von «endogenen» bzw. inneren Ursachen ausgehen (NB: dabei ebenfalls den Descartes'schen Dualismus akzeptieren, genau wie der zellularpathologische Krankheitsbegriff ja auch). Hahnemann hat mit seinem Ansatz den Descartes'schen Dualismus (übrigens ein Kind der Inquisition) überwunden; leider haben etliche seiner Epigonen dies nicht verstanden und die innere Ursache von Erkrankungen, im Gegensatz zur äusseren Hahnemanns wieder in die Homöopathie eingeführt.

Für homöopathische Ärzte: Der bedeutendste aberrierende Vertreter (und mit ihm alle seine Nachfahren) war J. T. Kent, bei dem zu lesen ist, «dass die innere, wahre Ursache jeder Krankheit in der Ursünde des Menschen, im aberrierenden Denken und Wollen eines Patienten zu suchen sei. Dass die Bakterien nicht die Erreger, sondern die Folge der Krankheit, die Leichenbestatter seien; dass darum die Aufgabe eines Arztes nicht darin bestünde, im Wasser, in ungesunden Wohnungen oder der Nahrung nach der Ursache einer Erkrankung zu suchen, sondern darin, im Innern des Menschen Ordnung zu schaffen.» [4] Vgl. dazu auch [8-10].
Wer eine «innere» Ursache bei Erkrankungen akzeptiert, öffnet der Spekulation Tür und Tor, und im Reiche der Spekulation ist bekanntlich jeder König.

Die Homöopathie arbeitet somit mit empirischen Regeln und Modellvorstellungen - genau wie die "Schulmedizin" auch. Wer demnach glaubt, die Homöopathie als Ganzes widerlegen zu können, indem er eine einzige Regel glaubt widerlegen $\mathrm{zu}$ können (meist erfolgen die Angriffe auf die von beiden Seiten nicht verstandene Verdünnungslehre), erliegt einem Irrtum. Ebenso wer glaubt, die Homöopathie sei deshalb widerlegt, weil einige unkritische Epigonen leicht $\mathrm{zu}$ widerlegende eigene Theorien verbreiten, und es gelingt, diese als unsinnig zu entlarven. Wer die Homöopathie verifizieren bzw. falsifizieren will, muss sich mit dem Gedankengut und den Originalwerken Hahnemanns auseinandersetzen. Tut man dies, so merkt man sehr rasch, dass Hahnemann ein durchaus wissenschaftlich denkender Kopf war und dass eine Diskussion zwischen kritischen homöopathischen Ärzten und kritischen Vertretern der Universitätsmedizin durchaus möglich ist. Dies bedingt jedoch, dass man erkannt hat, dass auch der Materialismus letztlich nur eine Arbeitshypothese ist und sich "Leben" naturwissenschaftlich nicht "erklären» lässt. So ist es verständlich, dass sich führende Kliniker wie Prof. Dr. med. Dr. h.c. mult. H. E. Bock, Tübingen, der Nobelpreisträger Prof. 0. Butenandt, der Pharmokologe Prof. Franz Gross, Heidelberg, in einer wissenschaftlichen Diskussion durchaus mit der authentisch interpretierten, d.h. therapiegeschichtlich fundierten Interpretation der Lehre Hahnemanns als wissenschaftliche Therapiemethode einverstanden erklären konnten [11; Briefwechsel und Literatur beim Verfasser erhältlich]. Nur so ist auch verständlich, dass in den Jahren um 1980 enge und fruchtbare Beziehungen zwischen dem "Schweizerischen Verein homöopathischer Ärzte» und Vertretern der medizinischen Fakultät der Universität Bern bestanden und dass auf der Grundlage einer authentischen Interpretation vor den Studierenden der Medizin Vorlesungen über Homöopathie im grossen Hörsaal gehalten werden konnten.

"Um das richtige homöopathische Arzneimittel zu finden, gilt es die Gesamtheit der zusammengehörigen Symptome eines Krankheitszustandes (nicht ıdes Kranken) zu bestimmen und dasjenige Arzneimittel anzuwenden (immer nur eines gleichzeitig), welches die zugrundeliegende Krankheit kurativ zu behandeln vermag. Dieses Mittel ist nur aufgrund von Erfahrung zu bestimmen." [3]

Kommentar: Die Homöopathie ist demnach eine «Erfahrungsheilkunde». Gemeint ist damit allerdings nicht die subjektive und unsystematisch erworbene Erfahrung; Erfahrung muss überprüfbar, kommunizierbar und reproduzierbar sein, andernfalls die Erfahrungsträger die hahnemannsche Grundidee der empirischen Erfahrungsgewinnung verlassen. Dass sich Erfahrungen betreffend Wirksamkeit (homöopathischer) Medikamente allerdings nur dokumentieren lassen auf dem Boden eines tragfähigen, stets mit 
sich selbst identischem Krankheitsbegriffs (vgl. auch "namenfähige Krankheiten» bei Hahnemann) ist eine Selbstverständlichkeit [5, 11, 12].

Für homöopathische Ärzte: Weder theoretische Überlegungen noch Spekulationen, noch Computer, noch Pendeln oder ähnliche Verfahren sind notwendig, um das richtige Arzneimittel herauszufinden. Wenn solche Verfahren eingesetzt werden, hat dies mit der Methode Hahnemanns nichts, aber auch gar nichts zu tun. Es handelt sich dann um eine neue Methode, bei der jedoch etwas wissenschaftlich Unzulässiges getan wird, es wird nämlich auf einer ersten Arbeitshypothese (z.B. Pendeln oder Bioresonanz etc.) eine zweite Hypothese aufgebaut, dass es nämlich möglich sein soll, mittels dieser Methode das richtige Arzneimittel zur Behandlung eines Krankheitszustandes herauszufinden.

\section{Die Homöopathie in der Schweiz}

In der Schweiz stehen sich seit der Nachkriegszeit im grossen und ganzen zwei verschiedene Interpretationen der Homöopathie gegenüber: Die aus medizinhistorischer und therapiegeschichtlicher Sicht als authentisch geltende Interpretation der Lehre Hahnemanns (vgl. dazu Tab. 1) und eine sich an das Gedankengut der Naturheilkunde anlehnende Richtung, dessen bedeutendster Vertreter J. T. Kent war (vgl. dazu unter Stichwort "Naturheilkunde" in [3]).

Eine ausführliche Darstellung der Geschichtlichen Zusammenhänge findet sich in meinem Beitrag zur "Weltgeschichte der Homöopathie» [13].

\section{Lösungsvorschläge einst und jetzt oder "Welche Homöopathen braucht das Land?"}

Für homöopathische Ärzte: Von 1972 bis 1989 hatte sich der damalige Präsident des "Schweizerischen Vereins homöopathischer Ärzte», Dr. Walter Buschauer, intensiv um eine "unité de doctrine" bemüht, da die damalige Situation betreffend die "Reinheit der Lehre» ähnlich desolat war wie heute. Ein 1973 beschlossenes Programm umfasste folgende Punkte: 1. Die homöopathische Ärzteschaft muss sich auf eine gemeinsame Interpretation ihrer Lehren einigen; sie darf 2. nicht länger in elitärem, esoterischem Denken verharren, sondern muss 3. den Dialog mit der Wissenschaft und den Universitäten suchen. Um im Sinne der erwähnten Vorschläge $\mathrm{zu}$ einer allgemein verbindlichen Interpretation der Homöopathie zu kommen, wurden in den folgenden Jahren verschiedene Referenten $\mathrm{zu}$ Grundsatzvorträgen eingeladen. Die verschiedenen Interpretationen der Lehre Hahnemanns führten zu der Erkenntnis, dass eine "unité de doctrine" nur durch eine Rückbesinnung auf Hahnemanns erkenntnistheoretischen Standpunkt möglich wäre.
Man wandte sich in der Folge an den weltweit wohl besten Kenner der Geschichte der Homöopathie, an Dr. Heinz Henne (1923-1988), den ehemaligen Leiter der medizingeschichtlichen Forschungsstelle am Robert-Bosch-Krankenhaus in Stuttgart. Er galt dank seiner medizinhistorischen und pharmakologischen Kompetenz als erste Autorität in der Auslegung der Hahnemann-Originalschriften [13]. Die Zusammenarbeit mit dem Medizinhistoriker und homöopathisch tätigen Internisten Heinz Henne wurde in den folgenden Jahren zum entscheidenden Element in der weiteren Entwicklung der Homöopathie in der Schweiz [12].

Der langjährige Präsident des «Schweizerischen Vereins homöopathischer Ärzte» hat, wie bereits kurz erwähnt, in einer Vorlesung am Berner Inselspital 1982 (im Einvernehmen mit der Interdisziplinären Fachgruppe für Studienplanung) eine Homöopathievorlesung - in Rückbesinnung auf Hahnemann - gehalten; er hat sich dort auf dem Boden der authentischen Lehre Hahnemanns von Kents spiritualistischer Interpretation und allen seinen Nachfolgern distanziert und die Homöopathie als eine individualisierende (immunstimulierende) Therapie vorgetragen, die auf Hahnemanns (Psora-)Hypothese der infektiösen Ätiologie chronischer Krankheitszustände basiert und als induktiv-empirische Wissenschaft im Einzelfall jedesmal der Verifikation bedarf. Die Vorlesung fand ihren Niederschlag in seinem Lehrbuch [14], das von medizinhistorischer Seite (Prof. Schadewald) wie von pharmakologischer Seite (Prof. F. Gross) sehr anerkennend rezensiert wurde.

Für homöopathische Ärzte: Die Vorlesungen wurden während Jahren, zuletzt gemeinsam mit mir, fortgeführt. Die Universität tat sich schwer damit, einen offiziellen Lehrauftrag zu erteilen, nicht $\mathrm{zu}$ letzt wegen der rotierenden personellen Besetzung der zuständigen Instanzen. Dies trotz unserer Warnung, dass immer mehr Nicht-Ärzte aller Art in die Therapie drängten, immer mehr Laien Heilung bei ihnen suchten. Nachdem Buschauer im «Schweizerischen Verein homöopathischer Ärzte» bei der zunehmenden Anzahl der Anhänger der Kentschen "Philosophie» immer weniger Anerkennung für sein Bestreben erhielt, brach der Kontakt ab.

Unter der späteren Präsidentschaft wurde nicht mehr inhaltlich-argumentativ diskutiert, sondern mit Hilfe des Stimmvolkes politischer Druck ausgeübt, der zu Lehraufträgen für sogenannte Komplementärmediziner führte. 


\section{Tabelle 1}

Medizinhistorische bzw. therapiegeschichtliche von der Homöopathie ausgehende Neuerungen und Impulse.

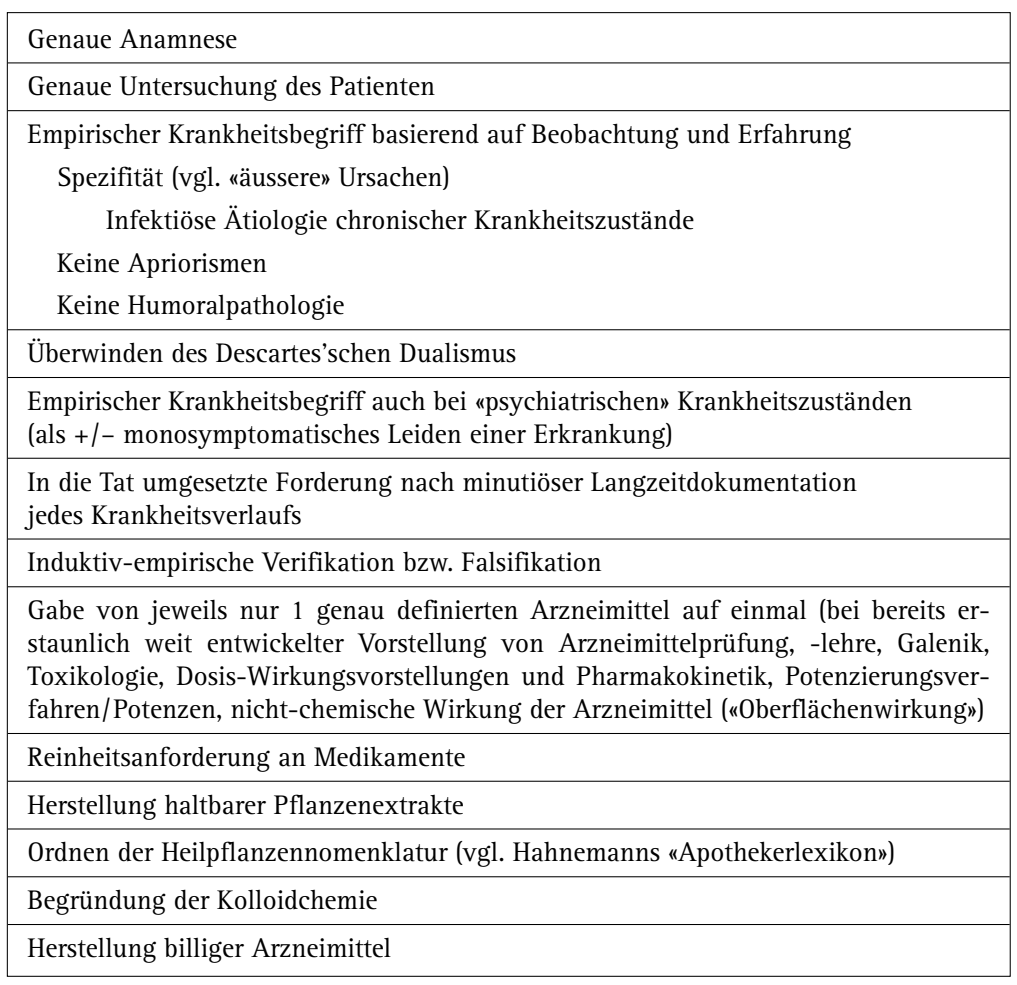

\section{Ausblick}

Seit kurzem ist die homöopathische Therapie nun eine Pflichtleistung der Krankenkassen. Dieser Zustand ist auf einige Jahre begrenzt, und ich sehe nicht, wie es der schweizerischen Homöopathie bis 2004 gelingen könnte, bei der herrschenden Orientierungslosigkeit, den Nachweis der Wirkung bzw. Wirksamkeit der Homöopathie zu erbringen. Was der Homöopathie in über 200 Jahren bis jetzt nicht gelungen ist, wird ihr kaum in vier bis fünf Jahren möglich sein, zumal noch nicht einmal eine "unité de doctrine» akzeptiert ist und die von einer nicht unerheblichen Anzahl homöopathischer Ärzte heute betriebene Homöopathie einer kritischen Analyse nicht standhält.

Für homöopathische Ärzte: Alle bis jetzt bekanntgewordenen Versuche in dieser Richtung scheinen mir ungeeignet. Gefordert wird bekanntlich der Nachweis von Wirksamkeit, Zweckmässigkeit und Wirtschaftlichkeit. "Wirksamkeit» darf nicht mit "Wirkungen" verwechselt werden; der experimentelle "Versuch" der Pharmakologie kann letztlich nur Wirkungen, nicht aber Wirksamkeit verifizieren, so dass die Kritik am experimentellen Vorgehen der modernen Medizin berechtigt erscheint. Die "Wirksamkeit» einer Therapie lässt sich nur mit dem "Hahnemannschen Versuchstyp" einer Langzeitdokumentation (viele Verläufe über eine lange Zeit) bewerkstelligen. Dasselbe gilt für "Zweckmässigkeit» und "Wirt- schaftlichkeit». Der «Schweizerische Verein homöopathischer Ärzte» steht zudem vor dem Problem, dass eine Anzahl seiner Mitglieder eine ungenügende schulmedizinische Ausbildung haben, kaum mehr sauber dokumentierte Diagnosen stellen können und wollen und das Verschwinden von Symptomen auch kaum objektiv belegen.

Fragebogen, welche das subjektive Wohlbefinden vor und nach homöopathischer Behandlung erfragen sollen, sind zwar interessant, werden bei kritischen Ärzten jedoch nur ein freundliches Lächeln hervorrufen, wenn es dereinst um die Anerkennung gehen wird. Um eigene Studien durchzuführen, sei es prospektiv krankheitsbezogen (bzw. diagnosebezogen, wobei nur "namenfähige Krankheiten" nach Hahnemann dazu geeignet sind) oder durch die Analyse bereits bestehender Fälle, genügt die Zeit wahrscheinlich nicht.

Es ist auch eher unwahrscheinlich, dass die Krankenkassen bzw. das Bundesamt für Sozialversicherung bereit sein werden, ihre Statistiken zur Verfügung zu stellen, um wenigstens den Aspekt der Wirtschaftlichkeit näher betrachten zu können; methodisch gesehen wäre dieses Vorgehen insofern fragwürdig, als aus einer solchen Kostenanalyse natürlich nicht hervorgehen würde, ob "spezielle» Patienten in den homöopathischen Praxen (d.h. "gesündere" Patienten, welche nur an Funktionsstörungen leiden) behandelt werden oder nicht.

Der Versuch, eine Anerkennung der Homöopathie auf demokratischem Weg zu erlangen (über Volksinitiativen, Petitionen, eine "Abstimmung mit den Füssen", d.h. der Forderung, es solle durch die soziale Grundversicherung das bezahlt werden, was die Patienten fordern, welche damit belegen, dass sie sich dort behandeln lassen, wo Erfolge erzielt werden), kann bestenfalls von kurz dauerndem Erfolg sein, wie uns ein Blick in die Therapiegeschichte lehrt.

Es stellt sich die Frage, wer bereit ist, an einem erfolgversprechenden Konzept mitzuarbeiten. Bedingen würde dies, dass man sich auf Hahnemanns Lehre zurückbesinnt, der sich im Bemühen um Arzneimittelsicherheit als erster in Deutschland nicht nur theoretisch, sondern auch in der Praxis von allen Dogmen, Systemen, Kosmologien in der Medizin abwandte und sich auf Hippokrates, auf die antike Empirikerschule, d.h. auf Beobachtung und Erfahrung als einzig tragfähiger Grundlage einer wissenschaftlichen Arzneimitteltherapie, zurückbesann. Wissenschaftlich ist aus heutiger Sicht nicht dogmatisches, aprioristisches und ontologisches Denken, sondern "der methodische Gang, der Schritt für Schritt (induktiv - empirisch) auf dem Boden der Erfahrung zur Entscheidung drängt». [nn] - Analog der «Fortdauernden Prüfung der Arzneimittel» bei Franz Gross. Die Erkenntnis führt zur erneuten Forderung, wie bereits von Henne und Buschauer 1985 erhoben, ein "Zentrum für homöopathisches Schrifttum» zu schaffen, $\mathrm{zu}$ dessen laufender Vervollkommnung alle 
homöopathischen Ärzte (und nur Ärzte) beitragen können, die bereit sind, sich auf den erkenntnistheoretischen Standpunkt Hahnemanns zurückzubesinnen. Ich erhebe diese Forderung als ehemaliges Vorstandsmitglied des "Schweizerischen Vereins homöopathischer Ärzte» und als ehemaliger Co-Referent von Buschauer an den Vorlesungen in Bern, da ich in tiefer Sorge um die Homöopathie Hahnemanns bin. Gerade deshalb habe ich die Karikatur von ANNA in Heft 46, wie eingangs erwähnt, so treffend empfunden.

\section{Zusammenfassung}

Auch wenn aufgrund einiger Studien Wirkungen bzw. Wirksamkeit der homöopathischen Therapie vermutet werden können, stehen die schweizerischen homöopathischen Ärzte auf gesetzgeberischen Anstoss hin vor der schwierigen Aufgabe, innert weniger Jahre den Nachweis von Wirksamkeit, Zweckmässigkeit und Wirtschaftlichkeit ihrer Methode zu erbringen. Da eine "unité de doctrine» im Moment fehlt (obwohl erarbeitet, vgl. Tab. 1 und Literaturverzeichnis), muss als erstes in dieser Richtung hin gearbeitet werden auf der Grundlage einer authentischen Interpretation der Lehre Hahnemanns.

Die homöopathischen Ärzte müssen sich klarer als bisher von allem esoterischen Denken, unwissenschaftlichen Spekulationen und Nichtärzten aller Art abgrenzen. Es ist mit aller Kraft die Schaffung eines "Zentrums für homöopathisches Schrifttum» voranzutreiben, ist doch den Belegen von Wirkung und Wirksamkeit aus der Literatur (wie bis anhin bei der IKS zur Erlangung der Zulassung eines Arzneimittels üblich) erste Priorität zuzumessen. Aufgrund des bereits Erreichten (Studien, vorhandene Dokumentation) könnte die Homöopathie, wenn sie die obengenannten Forderungen erfüllt, darauf pochen, dass sie mit der gleichen Elle wie die Schulmedizin gemessen wird, d.h. sie kann ihrerseits fordern, dass auch die (innere) Medizin (bzw. die Allgemeinmedizin) für jede ihrer Handlungen den Nachweis von Wirksamkeit, Zweckmässigkeit und Wirtschaftlichkeit innert vier bis fünf Jahren erbringen muss. Da die Schulmedizin und die Homöopathie letztlich vor den gleichen Anforderungen stehen, wäre eine Zusammenarbeit im Interesse der Patienten und der Wissenschaft sehr wünschenswert.
Kollegen, die sich angesprochen fühlen, aufbauend auf den in Tabelle 1 dargestellten Errungenschaften Hahnemanns und der authentischen Interpretation seiner Lehre, die er Homöopathie genannt hat, an der Verifikation bzw. Falsifikation derselben mitzuarbeiten und darauf basierend wieder einen echten Dialog auf wissenschaftlicher Basis mit der Universität zu suchen, sind herzlich eingeladen, mit mir in Verbindung zu treten. Therapiegeschichtliche Unterlagen bzw. Quellenstudien sind reichlich vorhanden.

\section{Literatur}

1 Hartmann A. Hahnemanns Homöopathie. [Karikatur]. Schweiz Ärztezeitung 1999;80(46):2691.

2 Holzapfel K. Kreuzfeuer oder Kreuzigung. KH 1998; 42(3):120-3.

3 Schweizer Lexikon in 6 Bänden. Luzern: Verlag Schweizer Lexikon, Mengis und Ziehr; 1992. Bd. 3, S.472-3.

4 Buschauer W. Homöopathie und Homöopathen. Heidelberg: Karl F. Haug Verlag; 1991.

5 Henne H. Quellenstudien über Samuel Hahnemanns Denken und Wirken als Arzt. Stuttgart: Hippokrates Verlag; 1963.

6 Remy M. Bericht über den 1. Internationalen HomöopathieKongress für Chronische Krankheiten, Frankfurt 11.-13. September 1998. KH 1998;42(6):258-9.

7 Jahr GHG. Die Lehren und Grundsätze der gesamten theoretischen und praktischen homöopathischen Heilkunst. Stuttgart: Verlag von Samuel Gottlieb Liesching; 1857. Nachdruck Ulrich Burgdorf Verlag für homöopathische Literatur.

8 Hehr GS. Bakteriologie und Homöopathie. The British Homoeopathic Journal 1982;71(2). Autorisierte Übersetzung.

9 Hehr GS. Was Kent a Hahnemannian? The British Homoeopathic Journal 1984;73(2):71-4.

10 Holzapfel K. Strömungen der gegenwärtigen Homöopathie. KH 1997;41(5):175-80.

11 Fäh L. Hahnemanns Lehre - Homöopathievorlesung im Berner Universitätsspital Bern 1987

12 Buschauer W. Zur authentischen Interpretation der Homöopathie als Vollendung der hippokratischen Medizin. Heidelberg: Karl F. Haug Verlag; 1985.

13 Fäh L. Späte Einheit in einem mehrsprachigen Land: Schweiz. In: Dinges M (Hrsg.). Weltgeschichte der Homöopathie; Länder, Schulen, Heilkundige. München: Verlag C. H. Beck; 1996. S. 102-17.

14 Buschauer W. Grundlagen und Praxis der Homöopathie. 2. überarbeitete Auflage. Heidelberg: Karl F. Haug Verlag; 1994. 